Case Report

\title{
From Eye Drops to ICU, a Case Report of Three Side Effects of Ophthalmic Timolol Maleate in the Same Patient
}

\author{
Muhammad Asim Rana, ${ }^{1}$ Ahmed Fouad Mady, ${ }^{2}$ Basheer Abdel Rehman, ${ }^{2}$ \\ Abdulrahman Alharthy, ${ }^{2}$ Basim Huwait, ${ }^{2}$ Asim Riaz, ${ }^{1}$ and Waleed Tharwat Aletreby ${ }^{2}$ \\ ${ }^{1}$ King's Mill Hospital, Nottinghamshire NG17 4JL, UK \\ ${ }^{2}$ Department of Intensive Care Medicine, King Saud Medical City, Riyadh 11373, Saudi Arabia
}

Correspondence should be addressed to Muhammad Asim Rana; drasimrana@yahoo.com

Received 11 May 2015; Accepted 28 July 2015

Academic Editor: Chiara Lazzeri

Copyright (C) 2015 Muhammad Asim Rana et al. This is an open access article distributed under the Creative Commons Attribution License, which permits unrestricted use, distribution, and reproduction in any medium, provided the original work is properly cited.

\begin{abstract}
Timolol Maleate (also called Timolol) is a nonselective beta-adrenergic blocker and a class II antiarrhythmic drug, which is used to treat intraocular hypertension. It has been reported to cause systemic side effects especially in elderly patients with other comorbidities. These side effects are due to systemic absorption of the drug and it is known that Timolol is measurable in the serum following ophthalmic use. Chances of life threatening side effects increase if these are coprescribed with other cardiodepressant drugs like calcium channel or systemic beta blockers. We report a case where an elderly patient was admitted with three side effects of Timolol and his condition required ICU admission with mechanical ventilation and temporary transvenous pacing. The case emphasizes the need of raising awareness among physicians of such medications about the potential side effects and drug interactions. A close liaison among patient's physicians is suggested.
\end{abstract}

\section{Case Report}

An 84-year-old male patient with past medical history consisting of hypertension, hypercholesterolemia, type 2 diabetes mellitus, and open angle glaucoma was admitted to ICU via A \& E with an unwitnessed collapse and decreased level of consciousness. In emergency department (ED) his GCS was recorded as $5 / 15$, blood sugar was found to be only $34 \mathrm{mg} / \mathrm{dL}$, his heart rate was 34 beats per minutes (bpm), and BP was 58/43 mm Hg. His ECG showed sinus bradycardia with variable blocks including sinus node dysfunction and type 1 Mobitz heart block pattern (Figures 1 and 2). He was intubated and ventilated in ED and given dextrose 50\% $100 \mathrm{~mL}$ and $0.5 \mathrm{mg}$ Atropine. His heart rate rose to $88 \mathrm{bpm}$ and with improvement of heart rate and correction of blood sugar his BP became 135/88 and his GCS improved to 13/15. Unfortunately, heart rate started to drop again to $40 \mathrm{~s}$ and blood sugar showed a downward trend for which he was given $1 \mathrm{mg}$ glucagon and was transferred to ICU. By the time he reached ICU his heart rate had improved to $90 \mathrm{bpm}$. In ICU after 30 minutes his heart rate dropped again so he was given another dose of Atropine and a temporary transvenous pace maker (TPM) was inserted. The patient's blood sugar also improved temporarily but eventually required intravenous infusion of $10 \%$ dextrose for the next 12 hours before it was stabilized. His GCS improved to $14 / 15$ and he was extubated the next day 16 hours after admission but repeated attempts to switch off his pace maker revealed underlying bradyarrythmias with heart rate dropping to $34-40 \mathrm{bpm}$ associated with presyncopal symptoms comprising of deterioration in attention and episodes of drowsiness. The pace maker was eventually switched off after 26 hours of observation when no more episodes of bradycardia were observed.

A detailed neurological assessment was carried out after extubation which showed the patient to be confused with no motor deficit. His CT brain was done which showed no abnormality and a detailed history was sought from the patient's wife who pointed out that the patient has been behaving in a weird and confused way for the last 5 days but his wife attributed that to demise of one of his close relative who died a week ago. She further added that he stopped taking his oral medications 2 days before he was admitted 


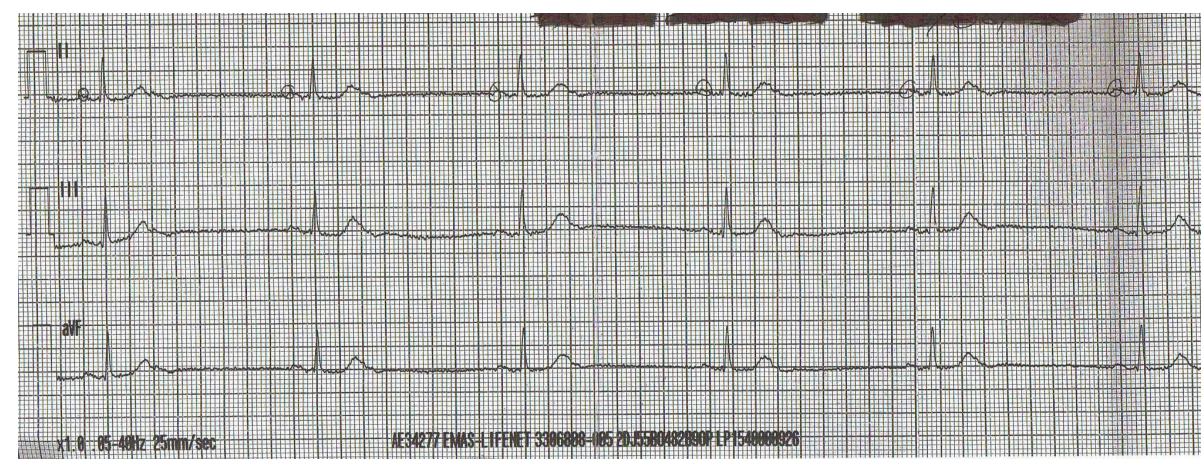

FIGURE 1: ECG showing sinus bradycardia.

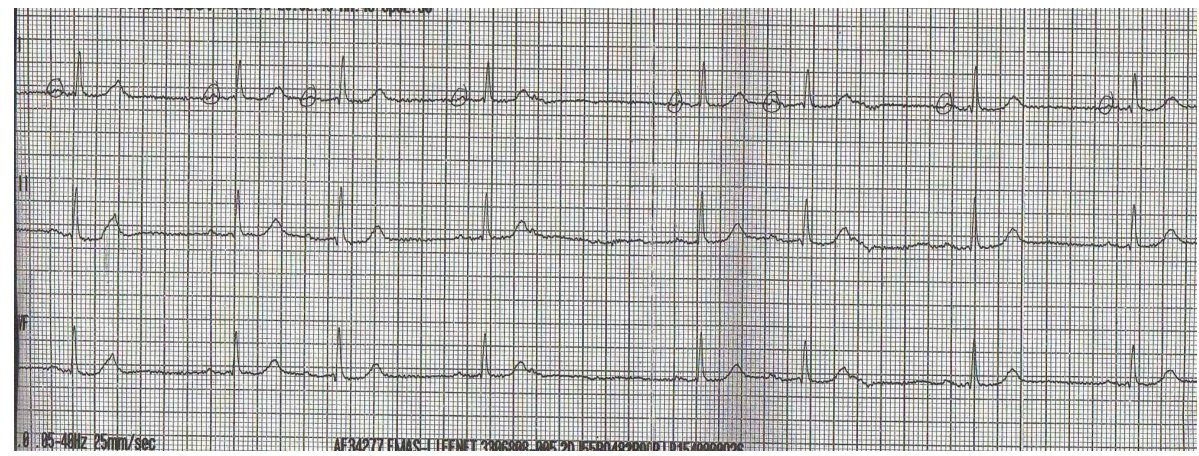

FIGURE 2: ECG with sinus dysfunction and Mobitz 1 block.

but he was given his eye drops regularly by his daughter who feared blindness in case the medication was not given. His drug history consisted of Enalapril $10 \mathrm{mg}$ OD, Atorvastatin $10 \mathrm{mg}$ OD, Metformin $500 \mathrm{mg}$ BID, and Timolol Maleate $0.5 \%$ one drop each eye twice a day which was started 35 days before admission to ICU when he was diagnosed as a case of glaucoma.

Tilt table test was not carried out and keeping in view his improvement in blood sugar and heart rate after administration of glucagon his clinical picture of hypoglycemia, confusion, and bradyarrhythmia was recognized to be because of ophthalmic Timolol Maleate which was changed to Travoprost (a prostaglandin F2 analogue) after consulting the ophthalmologist.

The patient was kept under observation in ICU for another 24 hours. His intrinsic heart rate improved to $82 \mathrm{bpm}$ and his TPM wire was removed before transferring to ward. Patient's confusion also improved gradually over the next 3 days and he was discharged on Enalapril, Travoprost, and Metformin. His followup for the next two months was unremarkable.

\section{Discussion}

Timolol was first introduced in 1978. It is a nonselective beta-adrenergic antagonist. It blocks both betal and beta2 adrenergic receptors. In the eye drop form, it has been the main stay of treatment in glaucoma and raised intraocular pressure secondary to traumatic cataract in $80 \mathrm{~s}$ and $90 \mathrm{~s}$ [13].
In general, beta-adrenergic blocking agents reduce cardiac output both in healthy subjects and patients with heart diseases. In patients with severe impairment of myocardial function, beta-adrenergic receptor blocking agents may inhibit sympathetic stimulatory effect necessary to maintain adequate cardiac function. Pharmacokinetics and pharmacodynamics of Timolol Maleate have been studied extensively. When given orally, Timolol is well absorbed and undergoes considerable first pass metabolism by cytochrome P450 2D6 enzyme system (CYP 2D6). Plasma half-life of Timolol is approximately 4 hours and metabolites are primarily excreted in urine [4].

Once administrated topically in eyes, almost $80 \%$ of the drops are drained through nasolacrimal duct to the nose, where they are absorbed systematically through nasal mucosa and it should be noted that as there is no hepatic first pass metabolism, the absorbed drug behaves like intravenous dose. Timolol can reach systemic circulation through conjunctival vessels as well. Timolol reaches peak concentration within 1 hour of topical administration with an average plasma concentration between $0.46 \mathrm{ng} / \mathrm{mL}$ and $1.38 \mathrm{ng} / \mathrm{mL}$ $[4,5]$. A dose of one drop of $2.5-0.5 \%$ solution to each eye is equivalent to a $5-10 \mathrm{mg}$ oral dose exposing the patient to adrenergic beta blocking effects and these include effects on CNS, pulmonary, cardiovascular, and endocrine systems [4$10]$.

Many reports are available in literature regarding Timolol Maleate's effects on heart rate and respiratory system. Some of them are particularly on interaction between Timolol and other cardiac drugs like beta blockers and calcium channel 
blockers $[11,12]$. Others are those in which Timolol was the only cause found [13-17].

Although hypoglycemia or altered response to blood sugar control has been listed a few times in literature [1719], topical beta blocker induced neurological side effects like confusion and psychosis are rare [20].

Our case report is unique in this regard where our patient developed three important side effects simultaneously and all of them improved after stopping the drug.

As pointed out earlier, cardiovascular effects have so far been extensively studied and reported [21, 22] and these include randomized control trials, crossover studies, or case reports but one thing should be noted that in most of these studies the participants included were healthy adults and they could not represent the real world patients which mostly consist of elderly patients with a number of comorbidities and most of them are already on either respiratory or cardiovascular medications. Moreover, these studies were limited to investigating the impact of Timolol Maleate in resting and peak heart rate during exercise but more serious side effects or outcomes like bradycardia or heart blocks were not assessed [23-26].

There is no specific antidote available for Timolol toxicity although there are some recommendations about the use of Glucagon 5-15 mg in slow intravenous infusion form if the patient does not respond to intravenous fluids and Atropine. In elderly patients, the effect of beta blockade has been found to be stronger and lasts longer [27].

Another important study was carried out by Pratt et al. [28] in which the researchers have looked at the association between ophthalmic Timolol and hospitalisation for bradycardia. This study summarizes some characteristics of patients admitted to hospital after initiation of Timolol. The major characteristics identified were mean age 82.6 and male sex preponderance $59.9 \%$. It was also pointed out that the risk of symptomatic bradycardia was significantly increased in the 31st to 180 days after Timolol initiation.

\section{Conclusion}

Topically administrated beta blockers could have profound and prolonged systemic side effects especially in older age group of patients. Extra caution should be observed when prescribing glaucoma treatment to these patients and careful medical and drug history should be sought. If they are found to have other respiratory or cardiovascular comorbidities like ischemic heart disease or COPD, then glaucoma regimen should be carefully chosen. A close liaison between patient's physician and ophthalmologist is therefore necessary. A small dialogue among the treating physicians can save the patient from a potentially serious and life threatening situation. Acute care physicians including those in emergency and ICU should keep their angle of suspicion wide and if such patients are admitted with syncope or falls, systemic adverse drug reaction should be considered.

\section{Conflict of Interests}

The authors have no conflict of interests do declare.

\section{References}

[1] T. J. Zimmerman and H. E. Kaufman, "Timolol: a $\beta$-adrenergic blocking agent for the treatment of glaucoma," Archives of Ophthalmology, vol. 95, no. 4, pp. 601-604, 1977.

[2] R. L. Radius, G. R. Diamond, I. P. Pollack, and M. E. Langham, "Timolol: a new drug for management of chronic simple glaucoma," Archives of Ophthalmology, vol. 96, no. 6, pp. 10031008, 1978.

[3] G. D. Novack, M. J. O’Donnell, and D. W. Molloy, “New glaucoma medications in the geriatric population: efficacy and safety," Journal of the American Geriatrics Society, vol. 50, no. 5, pp. 956-962, 2002.

[4] T. Nieminen, T. Lehtimäki, J. Mäenpää, A. Ropo, H. Uusitalo, and M. Kähönen, "Ophthalmic timolol: plasma concentration and systemic cardiopulmonary effects," Scandinavian Journal of Clinical \& Laboratory Investigation, vol. 67, no. 2, pp. 237-245, 2007.

[5] J. W. Shell, "Pharmacokinetics of topically applied ophthalmic drugs," Survey of Ophthalmology, vol. 26, no. 4, pp. 207-218, 1982.

[6] J.-M. Korte, T. Kaila, and K. M. Saari, "Systemic bioavailability and cardiopulmonary effects of $0.5 \%$ timolol eyedrops," Graefe's Archive for Clinical and Experimental Ophthalmology, vol. 240, no. 6, pp. 430-435, 2002.

[7] M. B. Affrime, D. T. Lowenthal, J. A. Tobert et al., "Dynamics and kinetics of ophthalmic timolol," Clinical Pharmacology and Therapeutics, vol. 27, no. 4, pp. 471-477, 1980.

[8] G. Alvan, B. Calissendorff, P. Seideman, K. Widmark, and G. Widmark, "Absorption of ocular timolol," Clinical Pharmacokinetics, vol. 5, no. 1, pp. 95-100, 1980.

[9] M.-L. Vuori, T. Ali-Melkkila, T. Kaila, E. Iisalo, and K. M. Saari, "Plasma and aqueous humour concentrations and systemic effects of topical betaxolol and timolol in man," Acta Ophthalmologica, vol. 71, no. 2, pp. 201-206, 1993.

[10] T. Nieminen, H. Uusitalo, V. Turjanmaa et al., "Association between low plasma levels of ophthalmic timolol and haemodynamics in glaucoma patients," European Journal of Clinical Pharmacology, vol. 61, no. 5-6, pp. 369-374, 2005.

[11] H. Uusitalo, J. Niño, K. Tahvanainen et al., "Efficacy and systemic side-effects of topical $0.5 \%$ timolol aqueous solution and $0.1 \%$ timolol hydrogel," Acta Ophthalmologica Scandinavica, vol. 83, no. 6, pp. 723-728, 2005.

[12] T. Minish and A. Herd, "Symptomatic bradycardia secondary to interaction between topical timolol maleate, verapamil, and flecainide: a case report," The Journal of Emergency Medicine, vol. 22, no. 3, pp. 247-249, 2002.

[13] J. A. Linkewich and I. M. Herling, "Bradycardia and congestive heart failure associated with ocular timolol maleate," American Journal of Hospital Pharmacy, vol. 38, no. 5, pp. 699-701, 1981.

[14] M. Anguita, F. Torres, D. Gimenez et al., "Bradyarrythmias secondary to the use of ophthalmic timolol. Areport of 3 cases," Revista Española de Cardiología, vol. 45, no. 1, pp. 71-73, 1992 (Spanish).

[15] M. Sharifi, J. M. Koch, R. J. Steele, D. Adler, V. J. Pompili, and J. Sopko, "Third degree AV block due to ophthalmic timolol solution," International Journal of Cardiology, vol. 80, no. 2-3, pp. 257-259, 2001.

[16] D. S. Prince and N. H. Carliner, "Respiratory arrest following first dose of timolol ophthalmic solution," Chest, vol. 84, no. 5, pp. 640-641, 1983. 
[17] W. L. Nelson, F. T. Fraunfelder, J. M. Sills, J. B. Arrowsmith, and J. N. Kuritsky, "Adverse respiratory and cardiovascular events attributed to timolol ophthalmic solution, 1978-1985," The American Journal of Ophthalmology, vol. 102, no. 5, pp. 606611, 1986.

[18] T. M. Velde and F. E. Kaiser, "Ophthalmic timolol treatment causing altered hypoglycemic response in a diabetic patient," Archives of Internal Medicine, vol. 143, no. 8, p. 1627, 1983.

[19] B.-Z. Silverstone and T. Marcus, "Hypoglycemia due to ophthalmic timolol in a diabetic," Harefuah, vol. 118, no. 12, pp. 693734, 1990.

[20] J. H. Shore, F. T. Fraunfelder, and S. M. Meyer, "Psychiatric side effects from topical ocular timolol, a beta-adrenergic blocker," Journal of Clinical Psychopharmacology, vol. 7, no. 4, pp. 264267, 1987.

[21] A. Waldock, J. Snape, and C. M. Graham, "Effects of glaucoma medications on the cardiorespiratory and intraocular pressure status of newly diagnosed glaucoma patients," British Journal of Ophthalmology, vol. 84, no. 7, pp. 710-713, 2000.

[22] C. V. Leier, N. D. Baker, and P. A. Weber, "Cardiovascular effects of ophthalmic timolol," Annals of Internal Medicine, vol. 104, no. 2, pp. 197-199, 1986.

[23] D. J. Brazier and S. E. Smith, "Ocular and cardiovascular response to topical carteolol $2 \%$ and timolol $0.5 \%$ in healthy volunteers," British Journal of Ophthalmology, vol. 72, no. 2, pp. 101-103, 1988.

[24] W. J. Doyle, P. A. Weber, and R. H. Meeks, "Effect of topical timolol maleate on exercise performance," Archives of Ophthalmology, vol. 102, no. 10, pp. 1517-1518, 1984.

[25] K. Dickstein and T. Aarsland, "Comparison of the effect of aqueous and gellan ophthalmic timolol on peak exercise performance in middle-aged men," American Journal of Ophthalmology, vol. 121, no. 4, pp. 367-371, 1996.

[26] W. C. Stewart, J. A. Stewart, S. Crockett, C. Kubilus, A. Brown, and N. Shams, "Comparison of the cardiovascular effects of unoprostone $0.15 \%$, timolol $0.5 \%$ and placebo in healthy adults during exercise using a treadmill test," Acta Ophthalmologica Scandinavica, vol. 80, no. 3, pp. 272-276, 2002.

[27] M.-L. Vuori and T. Kaila, "Plasma kinetics and antagonist activity of topical ocular timolol in elderly patients," Graefe's Archive for Clinical and Experimental Ophthalmology, vol. 233, no. 3, pp. 131-134, 1995.

[28] N. L. Pratt, E. N. Ramsay, L. M. K. Ellett, T. A. Nguyen, and E. E. Roughead, "Association between ophthalmic timolol and hospitalisation for bradycardia," Journal of Ophthalmology, vol. 2015, Article ID 567387, 6 pages, 2015. 


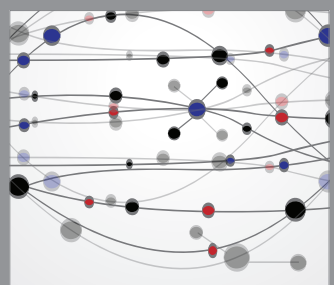

The Scientific World Journal
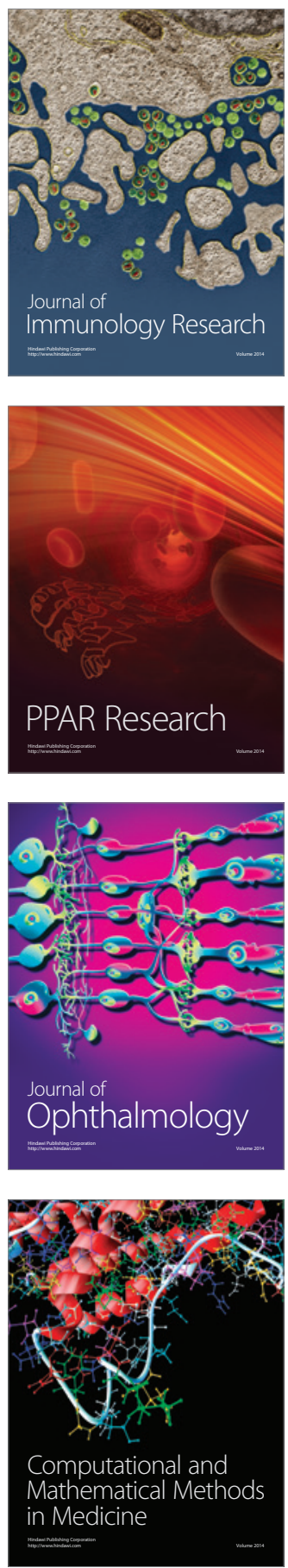

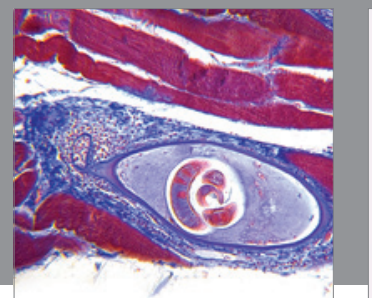

Gastroenterology

Research and Practice
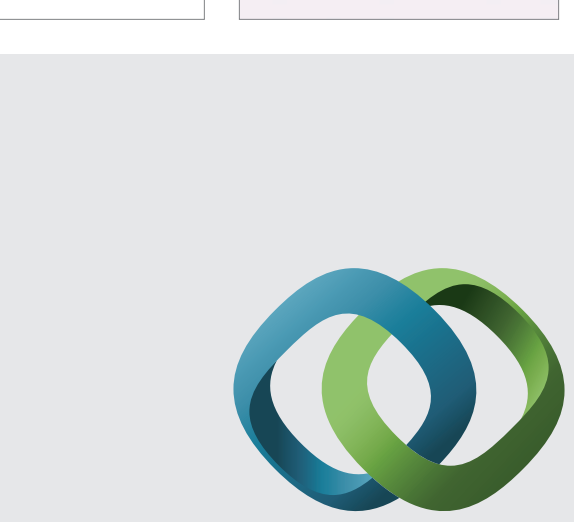

\section{Hindawi}

Submit your manuscripts at

http://www.hindawi.com
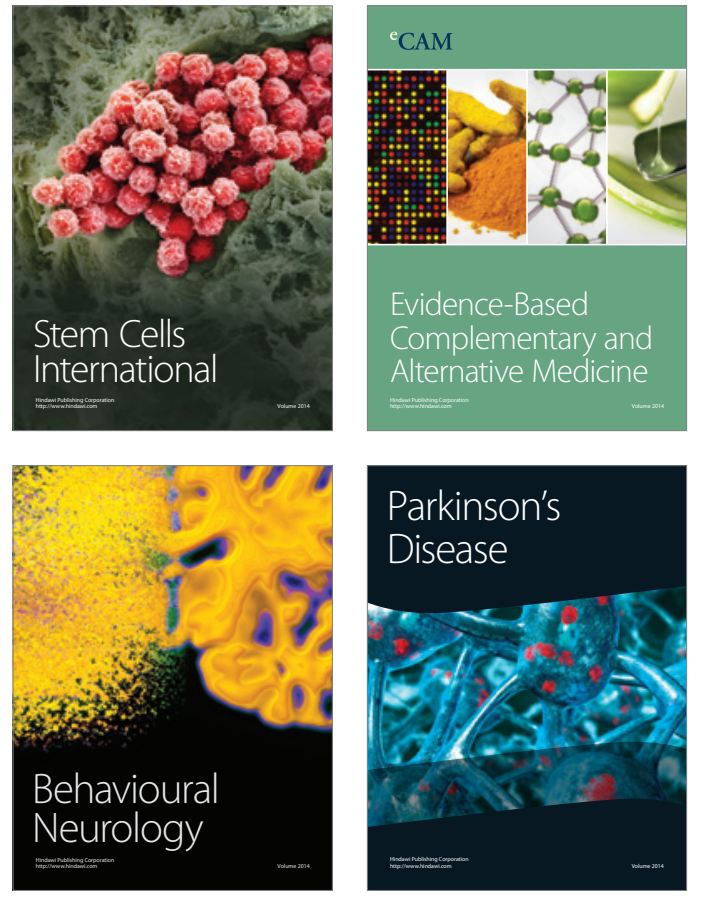
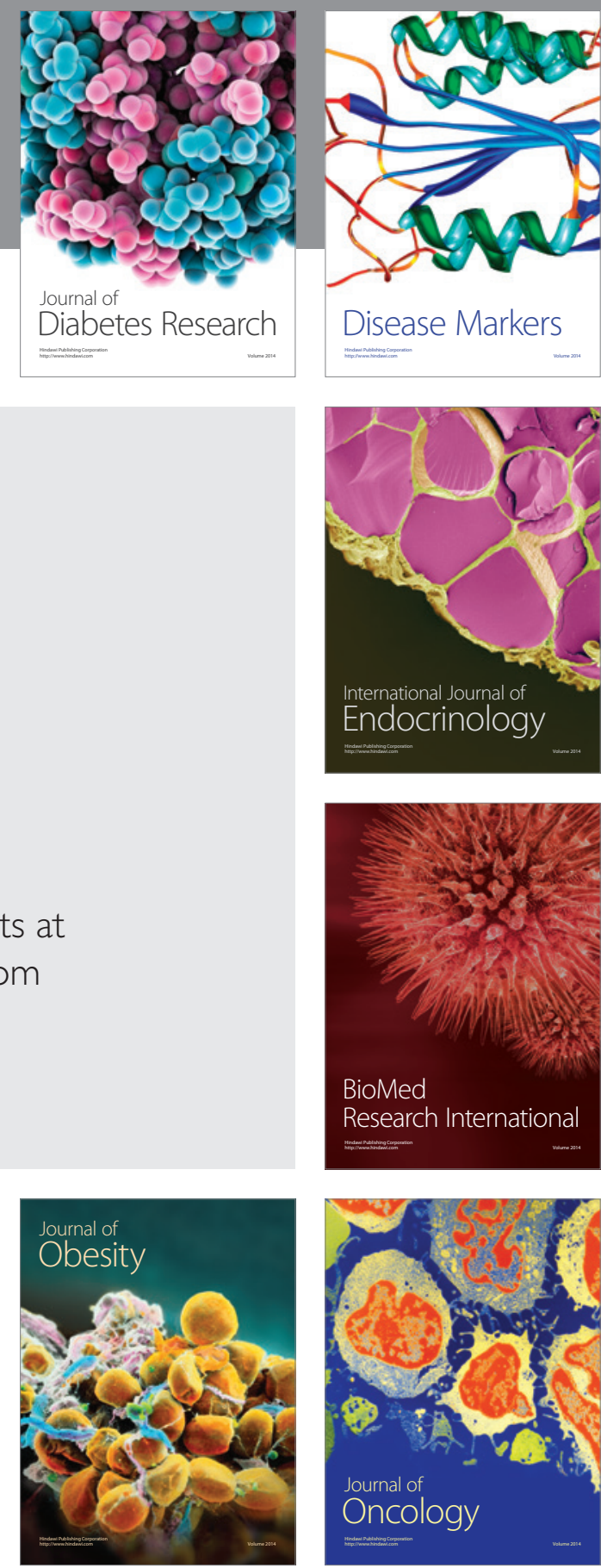

Disease Markers
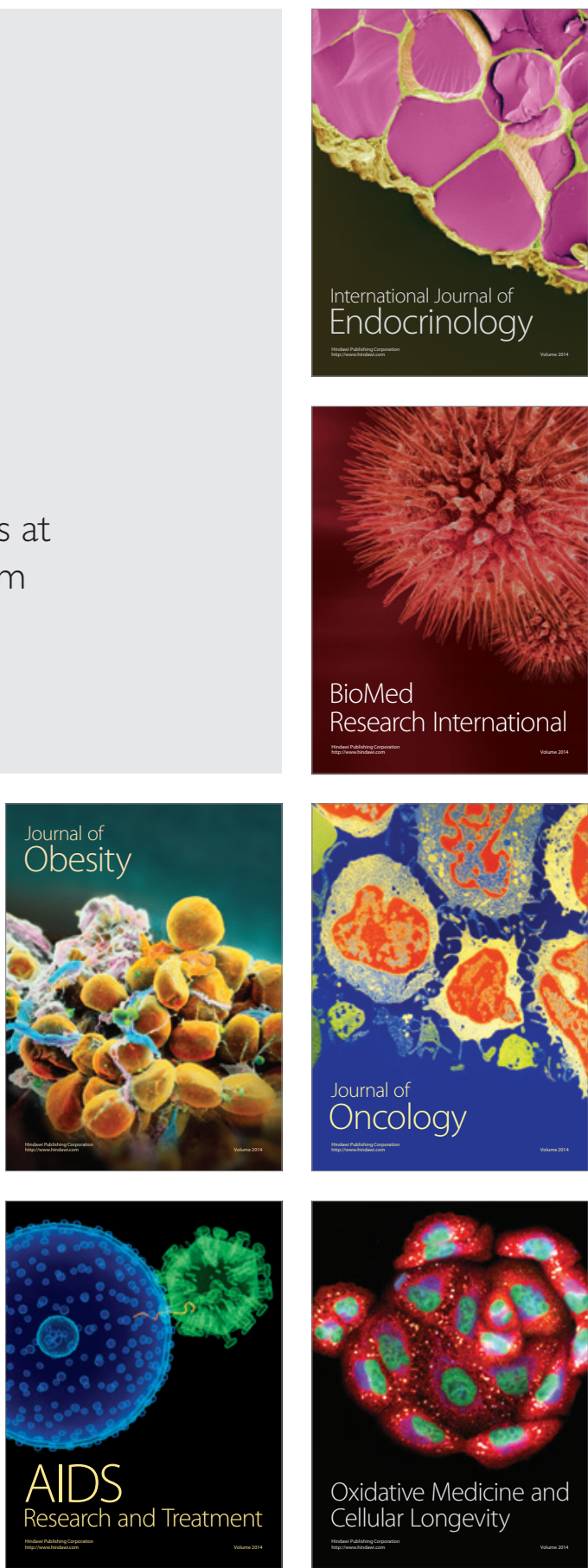